\title{
CHARACTERIZATION OF SEMIINSULATING GaAs: Cr BY SCANNING ELECTRON ACOUSTIC MICROSCOPY
}

\author{
B. MÉNDEZ and J. PIQUERAS
}

Departamento de Física de Materiales, Facultad de Físicas, Universidad Complutense, SP. 28040 Madrid, Spain

The intensity of the scanning electron acoustic microscopy (SEAM) signal in GaAs can show a marked dependence on the kind of material investigated. In doped semiconducting samples the electron-acoustic signal increases with the doping concentration (1) while in undoped semiinsulating material the relatively high signal level allows the observation of the defect distribution in the SEAM images (2). In the present work, the effect of doping level and electrical resistivity on the electron-acoustic response of different GaAs samples is investigated. It is found that SEAM is particularly useful to characterize semiinsulating GaAs:Cr due to the high electron-acoustic signal generated in this material.

The samples used were three n-type GaAs:Te wafers with n ranging from $2.2 \times 10^{17}$ to $1.5 \times 10^{18} \mathrm{~cm}^{-3}$, two semiinsulating GaAs:Cr wafers with $\mathrm{Cr}$ concentrations of about $5 \times 10^{15}$ and $5 \times 10^{16} \mathrm{~cm}^{-3}$ and an undoped semiinsulating GaAs wafer. The resistivity is higher for higher $\mathrm{Cr}$ concentration. SEAM measurements were performed in a Cambridge S4-10 and a Hitachi 2500 scanning electron microscopes. In order to get additional information about recombination processes, cathodoluminescence (CL) from GaAs:Cr samples was also measured in the electron microscope. CL from the other samples has been previously studied (2)(3).

The SEAM signal of GaAs:Te increases with dopant concentration. However, dislocations or dopant striations, detected by CL (3), are not observed in the SEAM images. The increase of the SEAM signal with $n$ would be related to the impurity induced change of thermal conductivity (1) and of the elastic constants. Compared with n-type GaAs, the SEAM signal of SI samples has been 
found to be about an order of magnitude higher and to increase with $\mathrm{Cr}$ doping. The typical cell structure of dislocations in SI GaAs is clearly revealed in the $\mathrm{Cr}$ doped samples with a structure which has not been observed in undoped SI GaAs (2). Fig. 1.a shows the linear (A(f)) SEAM image of a GaAs:Cr sample with a complex contrast related to the cell structure. The SEAM image of the same sample obtained in the non-linear mode (Fig. 1.b) shows dark cell walls. A contrast similar to that of Fig. 1.b is observed in the CL image revealing that a high linear SEAM signal corresponds to regions of enhanced non radiative recombination probability.

The differences in the electron acoustic behaviour of the GaAs samples can be explained by the relative contributions of different signal generation mechanisms. Thermoacoustic coupling can be related to the EA signal increase with concentration in GaAs:Te through the impurity induced lattice thermal conductivity changes but cannot explain other observations of the present work. In GaAs, electrostrictive and piezoelectric coupling are also present as EA signal generation mechanisms. The fact that in GaAs:Cr, CL and non-linear acoustic images are similar agrees with the existence of a contribution of excess carriers (electrostrictive coupling) to the acoustic signal. Furthermore, in seminsulating GaAs the piezoelectric coupling could represent the main electroacoustic mechanism. In SI material the strain fields at dislocation walls are clearly revealed in SEAM images while in GaAs:Te the high carrier concentration can screen the piezoelectric field providing no SEAM contrast.

a)

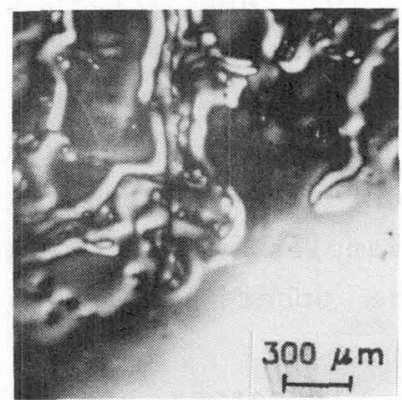

b)

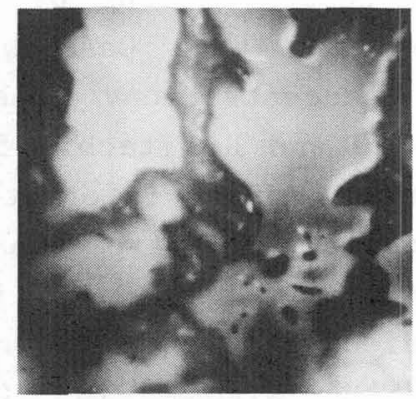

Fig. 1. a) Linear SEAM image of a GaAs:Cr sample.

b) Non linear SEAM image of the same region.

References

1) J.F. Bresse and A.C. Papadopoulo, J. Appl. Phys. 64, 98 (1988)

2) B. Méndez and J. Piqueras, Inst. Phys. Conf. Ser. No. 100, 789 (1989)

3) B. Méndez and J. Piqueras, J. Appl. Phys. 69, 2276 (1991 